\title{
Teorías subjetivas de profesores acerca de su rol en la construcción de la convivencia escolar
}

\author{
Gabriel Retuert Roe \\ Universidad de La Serena, Coquimbo, Chile. \\ Email: gretuert@userena.cl \\ Pablo J. Castro \\ Universidad de La Serena, Coquimbo, Chile. \\ Email: pablocastro@userena.cl
}

\begin{abstract}
Resumen $^{1}$ : Este artículo describe e interpreta las teorías subjetivas que elaboran los profesores de enseñanza media de Liceos de la ciudad de La Serena, acerca de su rol profesional en la construcción de convivencia escolar. Mediante un método cualitativo, se realiza un análisis de contenido con procedimientos de recolección y un análisis de la grounded theory. Con este propósito se aplican 4 entrevistas grupales, participando 30 docentes de 4 establecimientos. Algunos de resultados obtenidos evidencian que los docentes definen a sus alumnos como carentes y con diversas necesidades. Esto los llevaría a justificar la adopción de un rol con funciones diversas y a definir un tipo de relación con los estudiantes en el plano individual y grupal. Así también, explican las conductas al interior del aula y orientan sus procedimientos para el manejo de la disciplina. A partir de esto, se establecen dinámicas de convivencia escolar que se re-construyen continuamente y validan a través de los años de experiencia profesional.
\end{abstract}

Palabras claves: Rol del profesor, relación profesor-alumno, prácticapedagógica, docente de secundaria, convivencia escolar

\section{Subjective theories of teachers about their role in the construction of school life}

\begin{abstract}
This paper describes and gives an interpretation to the subjective theories that high school teachers of public schools of La Serena (Chile) produce about their professional role in the construction of school life. It is performed through a qualitative approach with a case study design, data collection, and grounded theory analysis procedure. With this objective in mind, 4 group interviews involving 30 teachers from 4 high schools have been carried out. Some of the results obtained show that teachers define their students as lacking and with diverse needs. This would lead them to justify the use of different functions in their pedagogical practice, and also define a relationship with the students in an individual or group perspective. Also, they explain the students' behavior within the classroom and how they guide their procedures for handling discipline. From this, school life dynamics are set and continually reconstructed, and they are subjectively validated by means of professional experience throughout the years.
\end{abstract}


Keywords: Teacher role, student/teacher relationship, teaching practice, secondary school teachers, school life

\title{
Teorias subjetivas de professores sobre o seu papel na construção da vida escolar
}

\begin{abstract}
Resumo: Este artigo descreve e interpreta teorias subjetivas que produzem professores do ensino secundário das escolas secundárias na cidade de La Serena, sobre o seu papel profissional na construção da vida escolar. Por um método qualitativo, realiza-se uma análise de conteúdo com procedimentos de recolha e uma análise da grounded theory. Para este efeito, aplicaram-se 4 entrevistas em grupo, participando 30 docentes de 4 estabelecimentos. Alguns dos resultados obtidos mostram que os professores definem os seus alunos como com carencias e necessidades diversas. Isto os levaria a justificar a adopção de um papel com várias funções e definir um tipo de relação com os alunos em nível individual e grupal. Além disso, explicar os comportamentos dentro da sala de aula e orientam os seus procedimentos para lidar com a disciplina. A partir disso, se estabelecem dinâmicas da vida escolar que continuamente são re-construídas e validadas através dos anos de experiência profissional.
\end{abstract}

Palavras-chave: Papel de professor, relação professor-aluno, prática de ensino, professor de ensino médio, vida escolar

\section{Introducción}

Los establecimientos educacionales hoy en día están inmersos dentro de un contexto social y cultural complejo. Una sociedad en gran medida impredecible por su dinamismo, donde opera la lógica del mercado en que todo se transforma en un bien de “consumo”, de vínculos humanos precarios, inestables y muchas veces superfluos y centrados en intereses personales. Un contexto sin pautas, en donde cada sujeto debe establecer sus propios modelos y sistemas de referencias y donde los medios de comunicación masiva buscan influir significativamente, creando irreales estándares de vida, sobredimensionando ciertos sucesos o personajes que "venden" o banalizando lo cultural, todos aspectos estudiados y que podrían constituir fenómenos de la post modernidad (Gergen, 2006; Bauman, 2003;Lash, 1999; Vásquez, 2008). Los centros educativos, no están ajenos a esto y como plantea Ianni (2003), las escuelas se forman a partir de la sociedad y al mismo tiempo expresan lo social, no quedando al margen de las crisis sociales, culturales y políticas. En este contexto, hablar de convivencia escolar ha ido cobrando progresivamente mayor significatividad, debido al tipo de problemáticas que se deben enfrentar como es la violencia escolar, el estrés laboral de los docentes, las dificultades de disciplina, entre otras dificultades que son de difícil manejo y contención y se constituyen en aspectos claves en la gestión escolar. En este marco, este estudio se aproxima al problema a través de las teorías subjetivas de los docentes, en cuanto a conocer sus explicaciones y significados acerca de su rol profesional en la 
construcción de la convivencia escolar y en el correspondiente estilo relacional que establecen con sus alumnos.

Entendiendo que la convivencia escolar es "la interrelación entre los diferentes miembros de un establecimiento educacional, que tiene incidencia significativa en el desarrollo ético, socio afectivo e intelectual de alumnos y alumnas" y que incluye las diferentes formas de interacción entre los estamentos que son parte de un establecimiento (Mineduc 2005), se torna particularmente relevante conocer los procesos subjetivos acerca de la construcción de la convivencia, siendo los profesores un foco de especial interés. El docente se constituye en un mediador entre el alumno y la cultura (Gimeno Sacristán, 1985) porque es quien traduce el currículum en función de los significados que atribuye tanto a los contenidos planteados del conocimiento en general, como de las actitudes que se organizan en torno a él (Aguilar, 2010). El papel que juega el profesor en torno a este proceso de construcción del convivir es fundamental, dado que en la forma en cómo éste entienda y ejerza su rol, posibilitará ciertos modelos y pautas de actuación social y afectiva entre profesores y alumnos.

La convivencia escolar es una construcción colectiva y dinámica que surge a partir de las diferentes interacciones al interior de un establecimiento educacional. Son diversos los factores que intervienen, los cuales no se reducen solo a lo meramente observable y explicito, sino que también a múltiples variables, relacionadas con el mayor o menor afecto en el trato interpersonal, como se gestiona la disciplina, las posibilidades que tienen los diferentes miembros de participar y expresarse, la apertura de la escuela a brindar espacios para actividades diversas de los alumnos, la acogida o rechazo que sientan tanto alumnos como apoderados a sus inquietudes, la formas y el criterio que se adopta para distribuir el poder, los estilos de trabajo entre profesores, entre otros factores vinculados al quehacer cotidiano de la escuela (Mena, Romagnoli y Valdés, 2006).

La convivencia constituye una trama compleja de diversas relaciones entre los actores que participan del proceso educativo de un establecimiento, constituyendo dinámicas de relación y poder que influyen en los climas emocionales, estilos comunicacionales y vinculares, que son propios del lugar donde se reproducen. Según Cassasus et al. (2001), el clima emocional del aula, esto es, lo que se constituye a partir de las relaciones sociales entre profesores y alumnos y entre alumnos, establece diferencias cuando se comparan dos escuelas similares pero con distintos resultados (Mena, Becerra y Castro, 2011). En este sentido, cuidar y velar por una convivencia que sea inclusiva, participativa, que facilite un clima emocional de respeto y valoración de las diferencias y que promueva una educación para la paz, puede constituirse en un factor preponderante para el desarrollo de una educación de calidad (Hirmas y Carranza, 2008).

Estudios demuestran que una buena convivencia escolar es un factor clave para el éxito de los aprendizajes y a la vez, contribuye a evitar y contener los problemas sociales que repercuten en los colegios y liceos 
(Donoso, 2005) “sin una formación humana que permita el normal desarrollo de la convivencia en la escuela y el interés por el conocimiento tampoco hay instrucción posible” (Asensio, 2004, p. 18). Algunas investigaciones muestran empíricamente esta relación entre los programas de educación en habilidades personales y sociales y su impacto en el aprendizaje y disciplina escolar; al respecto, evidenciando que programas educativos orientados al aprendizaje y que integran el desarrollo de habilidades socio afectivas y éticas como un componente importante en la formación escolar, tienen un impacto significativo en un mejor ambiente de trabajo, aprovechamiento de la vida escolar, aumento de los aprendizajes, incremento del rendimiento, mejora del apego escolar, mejor disciplina, salud mental y en general, una mayor desarrollo integral y formación ciudadana (Mena, Romagnoli y Váldes, 2009).

En el acercamiento al fenómeno de la convivencia escolar, una premisa importante se refiere a que a convivir se aprende en el espacio de interrelaciones, esto es, en la práctica misma de las relaciones humanas, lo que no está exento de dificultades que son propias de la vida humana y del encuentro con un otro u otros (Maldonado, 2004). Es un aprendizaje que se desarrolla en el tiempo y que surge y se construye en las dinámicas propias de las relaciones y negociación de significados tanto personales como compartidos. Por ello, los establecimientos que tienen la intención de generar dichos aprendizajes, tienen resultados en la formación de los alumnos que difieren de aquellos que no lo hacen o están centrados sólo en propósitos academicistas y basados en resultados. Desde esta perspectiva, Ruz (1997; Ruz y Bazán, 1998) plantea que las instituciones educativas actuales ante el panorama incierto de la educación, se encuentran en tensión producto de la necesidad de tener que lograr resultados, trabajo y progreso, y por otro lado, entregar formación, reflexión y educación. De acuerdo a estos autores, esto lleva a la imperiosa necesidad de propiciar nuevos modelos de práctica pedagógica centrados en la convivencia y la diversidad como una respuesta valórica, con un fuerte sentido formativo y transformador, ante las exigencias del contexto actual. Y agregan: "Esta mirada de la convivencia y la diversidad, supone admitir que las actuales prácticas pedagógicas, así como la sociedad en que ocurren, no pueden seguir como están y que se necesita un cambio” (Aristeguí, Bazán, Leiva, López, Muñoz y Ruz 2005, p. 140). Los profesores deberían tener más interés por métodos de enseñanza que les permitieran tener una mayor incidencia en otros aspectos de la educación que tienen un significado tanto para la vida personal como la comunitaria, antes que en "inconexos" saberes de cada disciplina (Aristeguíet al., 2005). En este marco, abordar la convivencia escolar conlleva, como propósito final

“...la dignificación de la persona humana, entendiendo que innovar para mejorar la convivencia equivale a posibilitar el desarrollo de competencias básicas para vivir la asociatividad, para manejar y asumir el conflicto, para lograr adecuados niveles de empoderamiento y de emancipación” (Arestegui, Bazán, Leiva, López, Muñoz y Ruz 2005, p. 5) 
Por otra parte, existen diversas problemáticas que derivan de las dificultades en la convivencia escolar, las cuales podrían clasificarse en tres grandes grupos: los actuales climas internos de aula, entendidos como los procesos de interacciones entre profesores y alumnos propios de la clase; la violencia que se visualiza en las interacciones de los actores escolares como el aumento percibido de fenómenos como el bullying; y el nivel de desgaste profesional actual que afecta a los docentes (Mena, Becerra y Castro, 2011).

Los roles se refieren a prescripciones en el comportamiento de una persona que ocupa una posición, es decir, pautas que ordenan sus acciones de acuerdo a la función que debe desempeñar. Definen relaciones recíprocas compuestas de derechos y deberes, con un otro u otros asociados o participantes (Hargreaves, 1979) y que dan sentido al lugar que ocupan, generando expectativas y comportamientos, según reglas de la comunicación e influencia social. Así también, se encuentra quien recibe el papel, compuesto de percepciones y cogniciones que aquel sujeto pueda tener acerca de lo que se le transmite o interprete del papel (Katz y Kahn, 1995). De acuerdo a Katz y Kahn (1995), una organización -como puede ser la escuela- se encarga de comunicar a cada uno de sus miembros los "sí" y los "no" asociados con el puesto que ocupen, pero será el papel percibido lo que de inmediato influirá en la conducta de la persona y se constituirá en fuente de su motivación y desempeño.

Tres enfoques que definen el rol docente: enfoque clásico centrado en el contenido, donde el profesor es principalmente el comunicador del saber, que presenta contenidos en forma expositiva y a través de demostraciones realizadas por él; enfoque centrado en las habilidades intelectuales, donde el alumno es protagonista y el profesor es quien guía u orienta el proceso de aprendizaje, proporcionando las condiciones necesarias para que este se logre, a la vez, el profesor diseña la enseñanza sobre la base de situaciones desconcertantes, sin una solución obvia, de manera que estimule la acción del alumno; y el enfoque, centrado en conocimientos y habilidades, como: contenidos culturales, destrezas, habilidades, actitudes y valores. Desde esta última perspectiva, el profesor asume como mediador entre el conocimiento específico y las comprensiones del alumno, a la vez, es facilitador de los aprendizajes y, finalmente, tiene un rol de investigador de los procesos del aula, en cuanto va reconstruyendo progresivamente su acción pedagógica para lograr aprendizajes significativos de sus alumnos (Pontificia Universidad Católica de Chile, 2006).

El presente estudio pone énfasis en las teorías subjetivas que realizan los profesores, y en particular a como éstos se explican y construyen significados en torno a la construcción de la convivencia escolar. De este modo, para facilitar la comprensión, se realizará una breve síntesis teórica acerca de las teorías subjetivas y conceptos relacionados. 
El ser humano, a partir de sus experiencias está permanentemente haciendo construcciones explicativas que le permitan ordenar y dar sentido a sus vivencias, de modo que le posibilite comprender los sucesos que le acontecen, para posteriormente orientar su conducta. Según Kelly (1955), cada sujeto tendría un sistema de construcciones que posee una organización cognitiva propia, y que le permite otorgar significado a lo que ocurre a su alrededor y desde allí, elaborar una determinada respuesta (Dallos, 1996). Estas construcciones estarían basadas en creencias, valores y conocimientos, que poseen un componente social y que influyen en la construcción de significados personales de los sujetos (Rodrigo, Rodríguez y Marrero, 1993). Algunos autores consideran que estos significados se van construyendo en forma recíproca con otros individuos, a partir de la participación en espacios de significados compartidos (Ascorra, Arias, y Graff, 2003).

Esta construcción de significados, puede concebirse como teorías personales, las cuales son representaciones individuales construidas desde las experiencias y que acontecen principalmente en entornos sociales. El proceso de construcción personal se encuentra influenciado por las pautas propias de la cultura y la interacción social en la sociedad, lo que da paso al modelo representacional que cada sujeto termina formándose (Rodrigo, Rodríguez y Marrero, 1993). De este modo, cada individuo reorganiza constantemente la información que recibe sobre la base de sus construcciones, ideologías y orientaciones de valor previamente construidas (Jones y Cooper, 1987; Kincheloe, 2001).

Para Rodrigo, Rodríguez y Marrero (1993), no es que un sujeto esté permanentemente categorizando su realidad, ya que esto, sólo se realizaría cuando es necesario; esto implica que las representaciones se activarían a través de metas o necesidades específicas del sistema, lo que ocurre de manera ocasional y de acuerdo a las circunstancias que la demandan.

El concepto de teorías subjetivas proviene de las explicaciones acerca de la construcción cognitiva de la realidad de Groeben y Scheele, quienes plantean una semejanza entre las explicaciones realizadas desde el mundo científico y las formas en que un individuo construye su propio conocimiento desde sus experiencias. Y define las teorías subjetivas como:

\footnotetext{
“Cogniciones de la visión de sí mismo y del mundo, que se pueden entender como un conjunto complejo, que tiene una estructura argumentativa, por lo menos implícita, y que cumple las funciones de explicación, predicción y tecnología, contenidas también en las teorías científicas”. (Groeben y Scheele citado por Avendaño, Krause y Winkler 1993, p. 109).
}

Desde la perspectiva de Flick (1992), estas teorías constituyen hipótesis, que surgen de la vida cotidiana y que los sujetos elaboran acerca de ellos y del mundo que les rodea, relacionadas entre sí por sus temáticas. Se pueden considerar "Teorías (o hipótesis) que elaboran los sujetos, para dar sentido a su entorno y para actuar en él. Estas elaboraciones del sujeto 
(subjetivas epistemológicamente) pueden ser tanto explícitas como implícitas”(Catalán, 2010, p. 22).

Flick (1992), plantea que las teorías subjetivas cumplen la función de orientar, en cuanto permiten enfrentar situaciones específicas; la función de justificar o de elaborar explicaciones a posteriori y la función de acomodar los propios sentimientos y valores. En definitiva, las teorías subjetivas permiten orientar la captación de la realidad; dar un marco explicativo a fenómenos y experiencias pasadas; predecir y sugerir nuevos comportamientos, lo que implica que guían el accionar del sujeto e involucran sentimientos de identidad que le dan un sentido subjetivo a la existencia del individuo (Catalán, 2010; Krause, 2005) y en particular para los profesionales, otorgar sentido a sus acciones (Castro Carrasco y Cárcamo, 2012).

Las construcciones elaboradas por el profesor, se enmarcan en el paradigma del pensamiento del profesor, entendido como el estudio de los procesos psicológicos básicos que ocurren en la mente de un profesor, quien organiza y orienta su comportamiento antes y durante la enseñanza y que tiene como fin, ayudar a la comprensión de cómo y por qué el proceso de enseñanza tiene la apariencia y el funcionamiento que lo caracteriza (Clark y Peterson, 1990; Gómez, 2008).

De modo particular, Marrero (1993) habla de teorías implícitas del profesorado, y las define como teorías personales pedagógicas que son reconstruidas sobre la base de los conocimientos pedagógicos elaborados históricamente y que son transmitidos ya sea a través de la formación como en la práctica educativa. Esta construcción constante del profesor, aún siendo personal, está directamente relacionada con el contexto próximo en la cual se realiza (Jiménez, 2005). En este sentido, es un proceso de permanente reconstrucción y diálogo con los espacios en que el sujeto de desenvuelve; así el profesor no puede pensar el currículum (propio de su rol pedagógico) fuera del contexto social donde se encuentra (Kincheloe, 2001).

En este contexto surge la presente investigación como una forma de conocer en profundidad las teorías subjetivas que elaboran los docentes que trabajan en liceos municipales de la ciudad de La Serena, Chile, acerca de su rol en la construcción cotidiana de la convivencia escolar. De este modo, se buscó conocer los procesos subjetivos de los profesores, accediendo a sus explicaciones en cuanto a su rol, para así lograr una mayor comprensión de sus lógicas de acción y visualización de sus alumnos. Por otra parte, comprender el nivel de implicancia subjetiva en los procesos formativos de sus alumnos, su percepción respecto de la relación profesoralumno y su incidencia en la construcción del clima de aula.

\section{Método}

Se utilizó metodología cualitativa desde un paradigma interpretativo (Vaschilachis de Gialdino, 1992). El estudio corresponde a una investiga- 
ción basada en la reconstrucción de teorías subjetivas de profesionales (Flick, 2009), en particular un grupo de docentes.Los procedimientos para la recolección y el análisis de los datos se basaron en los planteamientos de la grounded theory (Glaser y Strauss, 1967)que permite descubrir teorías, hipótesis y proposiciones desde los mismos datos o sujetos que los elaboran y no de supuestos a priori o concepciones teóricas existentes (Taylor y Bogdan,1996).

Se utilizó muestreo por conveniencia, que estuvo compuesta por profesores y profesoras de enseñanza media, como único criterio de inclusión se consideró que tuvieran un mínimo de tres años en el sistema escolar, que fuesen de diferentes asignaturas y que trabajaran al momento de la entrevista, en liceos pertenecientes a la Corporación Gabriel González Videla de La Serena. En el estudio participaron tres liceos técnico profesionales y uno científico humanista.Se realizaron cuatro entrevistas grupales (una por establecimiento) considerando la participación de 8 profesores por cada entrevista, realizadas en base a un guion temático semiestructurado. Se proponía un ámbito relacionado con las preguntas directrices de la investigación y los sujetos iban respondiendo y reflexionando acerca de lo consultado.

La muestra quedó constituida por 30 profesores (en dos de las entrevistas se ausentó un profesor), de cuatro establecimientos de enseñanza media y técnico profesional, que impartían clases en una de las siguientes asignaturas: lenguaje y comunicación, Historia y Ciencias Sociales, Biología, Inglés,Educación Artística, y Matemáticas; y en el caso de los liceos técnicos, se incluyó también a profesores de asignaturas de especialidad. El análisis de datos contempló el método de comparación constante(Glaser y Strauss, 1967), lo que implicó que en la medida que se fueron recogiendo los datos se fueron analizando inmediatamente en base a los objetivos y a partir de esto, se levantaron hipótesis que luego guiaban las siguientes entrevistas.

El proceso consistió en tres codificaciones: la codificación abierta, codificación axial y la codificación selectiva. La codificación abierta fue la fragmentación inicial de los datos en unidades descriptivas menores que permitieron ordenar y sistematizar la información en códigos, categorías y familias. La codificación axial, fue la identificación de dos grandes fenómenos centrales: Rol Docente Diverso y Convivencia Escolar, fruto del análisis y relación entre categorías y familias con un fin más interpretativo. Finalmente la codificación selectiva, denominada: Dinámica de Convivencia Escolar, reúne en un análisis final, todas las categorías desde una perspectiva más interpretativa y plantea una teoría explicativa que intenta responder al problema de investigación. Durante este proceso de codificaciones se alcanzó saturación teórica, en el sentido de que los datos que fueron emergiendo en nuevas entrevistas no continuaron agregando información nueva relevante (Krause, 1995).

Se mantuvieron presentes criterios de rigor para mantener el estándar científico, como: densidad, al realizar entrevistas grupales en profundidad, 
todas documentadas que permitió el análisis exhaustivo de cada entrevista; triangulación al momento de compartir resultados con otros investigadores en equipo, lo que permite también cumplir con el criterio de intersubjetividad; transferibilidad y aplicabilidad, al elaborar un modelo teórico acerca de las teorías subjetivas que permite conocer las explicaciones de los docentes acerca de su rol y así elaborar y dirigir posibles intervenciones en el ámbito de la convivencia.

\section{Resultados}

A partir de los procesos de análisis realizados, es posible plantear dos grandes modelos axiales que dan cuenta de interpretaciones compartidas por los docentes en su conjunto, dado que en el análisis no emergieron diferencias importantes en sus significados entre los grupos de entrevistados. Por un lado las teorías subjetivas relacionadas con un rol docente identificado como "diverso” y las teorías subjetivas acerca de la "construcción de la convivencia escolar” cotidiana y su impacto en la sala de clases. Un modelo explicativo final, que permite comprender y explicar para estos sujetos, como influye su mirada acerca de sus alumnos, del entorno y del sistema escolar en el cual se encuentran insertos, en las dinámicas cotidianas de construcción de convivencia.

\section{Teoría subjetiva acerca del rol docente diverso}

Los profesores expresan que sus alumnos poseen carencias diversas, que si bien se enmarcan desde un contexto social y un contexto familiar particular, la mayor parte posee carencias de tipo: afectivas y/o emocionales y valóricas, aunque éstas últimas están vinculadas sólo a algunos valores. Las primeras aluden a que los alumnos no cuentan en su mayoría con el apoyo y los cuidados necesarios por parte de sus padres y familiares, así como expresa una profesora: “...más con los niños de este, de este estrato social que están faltos de cariño, faltos de una persona que los escuche, faltos... con muchas carencias emocionales” (Entrevista -E2). Aspectos que dada la carga emocional de sus problemáticas familiares, los alumnos no podrían desprenderse de ellos.En relación a la carencia de valores, los profesores señalan que sus alumnos no tienen mayores referentes a los cuales seguir o que les inculque principios básicos, entre los cuales se encuentra fundamentalmente el "respeto" y que desde su perspectiva sería la base de otros.

De este modo se fundamenta una teoría subjetiva predominante, que se podría reconstruir como: “ya que...los alumnos poseen diversas carencias, entonces... se requiere un tipo de rol por parte del docente que permita enfrentar la mayor parte de estas necesidades”. Esta teoría se puede representar en el fenómeno central denominado como Rol Docente Diverso, el que entienden como una multiplicidad de acciones que deben ejercer en sus prácticas cotidianas de acuerdo al perfil de alumno con el cual trabajen. Desde esta perspectiva, los significados de los profesores en relación a su 
rol y sus funciones, pueden agruparse en: a) función mesiánica, salvadora o luchadora del rol, b) función de soporte afectivo que puede subdividirse en protectora, de contención y de suplencia parental, c) una función orientada hacia educar para la vida y d) la función de formación de valores para el trabajo. Las anteriores funciones son descritas por los docentes aportando diferentes matices que completan la teoría subjetiva predominante en torno al rol diverso, no siendo estos excluyentes entre sí.

La función mesiánica para un grupo de profesores, lo refieren a la “misión” de salvar o rescatar a sus alumnos desde el contexto del cual provienen, para así darles la posibilidad de que puedan surgir y ser personas valiosas para la sociedad. La mayoría alude a esto como una "lucha" constante que deben tranzar diariamente para poder "salvar" a estos alumnos y rescatarlos de su destino, "yo las miro y les digo no importa niñitas, paso una a una por una y las miro, se que una o dos se salvarán y esa será mi felicidad” (E2).

Un significado compartido por los docentes es considerar que estos aspectos los van sensibilizando en el tiempo [lo que les va otorgando experiencia docente] y esto explicaría, desde sus teorías, el por qué deben asumir y cumplir con funciones diversas en su acción profesional, y en particular también una labor orientada a ser soportes afectivos.

En este sentido, varios justifican que sus alumnos requieren de su cercanía afectiva, para poder contenerlos emocionalmente, valoraros, escucharlos y en algunas ocasiones "apretarlos" o sancionarlos cuando es necesario, una docente expresa: "te vas dando cuenta que las niñas no necesitan un apretón frío sino que ese apretón cálido (de afecto)“ (E1). Otra forma de expresión de este soporte afectivo, ocurre cuando se orienta suplir responsabilidades que son propias de las figuras parentales.

Por otra parte, hay quienes dicen tener una función más orientada a educar a sus alumnos para la vida, inculcándoles a temprana edad las responsabilidades y necesidades propias de una persona adulta, que debe trabajar, auto sustentarse y responder ante un grupo familiar. Para algunos docentes es tan relevante esto que incluso en un momento de la clase puede primar por sobre el aprendizaje de contenidos de una asignatura:

Una última agrupación de significados en torno al rol, tiene que ver con la función de ser educadores de valores, los cuales están principalmente relacionados con la vida laboral. Aun cuando la mayor parte de los profesores del estudio provenían de la educación técnico profesional; en el grupo de profesores pertenecientes a educación científico humanista, se aprecia una identificación similar hacia esta función formadora, aludiendo a valores similares, aunque es menos explícita la idea futura del trabajo.

Al asumir estas características de su rol, definen requisitos que los profesores deben tener para trabajar con alumnos de la educación pública, aludiendo que no cualquier profesor está capacitado para responder en 
forma adecuada ante las demandas del contexto. Los profesores expresan que un mayor dominio y habilidad para trabajar, sólo se va logrando con los años y con las múltiples experiencias vividas, lo que en el tiempo les permite tener una mayor flexibilidad para enfrentar dificultades propias del trabajo, mayor manejo disciplinario y comprensión de las problemáticas de los estudiantes. Esta mayor flexibilidad también les permite poder adaptarse mejor a las demandas del contexto escolar, por complejo que sea:

"entonces yo creo que estas cosas se van aprendiendo con la práctica, con los años de experiencia, con los casos especiales que uno va tendiendo en cada curso y los mismos alumnos te van formando a ti como profesor" (E1).

Otros requisitos que se sugiere a partir de sus teorías subjetivas, se refiere a: ser cercanos afectivamente y empáticos con los alumnos, logrando contener sus necesidades afectivas, comprender sus dificultades y transmitir preocupación y afecto. Ser coherentes y consecuentes entre sus acciones y discursos, dado que desde su perspectiva, les permite ser respetados y a la vez, educar en el respeto desde la acción: “cumplir, no quedarte en el discurso, sino que también llevarlo a la acción, desde ese punto de vista, ellos también aprenden a respetar la opinión porque saben que las cosas son como se dicen”. (E3). Y finalmente, poseer dominio pedagógico, entendido esto, como el manejo de los contenidos que imparte y la capacidad para manejar disciplinariamente al grupo de curso y en particular a los alumnos más conflictivos o difíciles.

Desde la diversidad de las funciones del rol sustentada por los profesores, surgen expectativas en cuanto al resultado del proceso formativo y en especial, de lo que ellos como docentes pueden generar en los estudiantes. La principal es que logren un mayor nivel de desarrollo personal y de posicionamiento social, siendo personas autónomas, con un proyecto de vida, maduros, con capacidad para formar familia, buenos ciudadanos y que puedan romper con el círculo de pobreza en la que muchos son parte.

Otras expectativas se refieren a que logren a futuro tener oportunidades laborales acordes con sus estudios (principalmente de tipo técnico), esperan que encuentren trabajo, que demuestren valores acordes con las exigencias laborales (respeto, puntualidad, responsabilidad) y que simplemente sean buenos trabajadores. A su vez, algunos esperan que sus estudiantes sean personas felices y que logren cumplir los sueños que tengan, a este tipo de expectativa se le ha denominado como una "visión romántica": "yo quiero que mis alumnas sean felices que sean más allá que profesional, que sean mujeres, que sean madres, preparadas para este mundo de hoy que es tan competitivo" (E1).

Los dos últimos conjuntos de expectativas, tienen que ver con el logro de valores y principios, relacionados con la integridad personal, esperando ellos que sean "personas de bien" y responsables. Finalmente, 
expectativas hacia la continuación de sus estudios superiores, aunque esta última agrupación, no es expresada en forma explícita por todos.

Como consecuencia de las diversas funciones de su rol y los requisitos para ser docente, los profesores explican que el rol del profesor recién egresado, tiene mayor dificultad, debido que no poseen el nivel de experiencia suficiente, son menos flexibles para enfrentar situaciones, les cuesta adaptarse y no conocen las características de sus alumnos, por lo que suelen ser distantes en su relación con ellos, por temor a la pérdida del control y dominio de grupo.

\section{Construcción de convivencia escolar}

Desde la perspectiva de los profesores, la convivencia escolar está influida permanentemente por una serie de factores, que pueden asociarse a tres ámbitos de influencia recíproca: “Institucional”, el “Alumno” y el "Profesor”. En los factores relacionados con lo institucional, los profesores señalan que el tipo de dirección del colegio y de gestión escolar por parte de las autoridades del colegio, influyen en el tipo de convivencia escolar que se desarrolle.

Así también, refieren que influye la organización interna, referido a las formas en que las personas se organizan y coordinan para llevar a cabo las diferentes funciones diarias. En los factores vinculados con el alumno, los profesores identifican por una parte aspectos asociados al contexto social de pertenencia. Otro factor importante sería la familia de origen de los alumnos, en términos de influencia en su formación, sus hábitos, valores y costumbres.

Así también, los docentes plantean que los alumnos poseen una “carga afectiva”, producto de sus experiencias familiares y sociales. Señalan que la mayor parte de sus alumnos, tienen diversas carencias afectivas, que surgirían por ausencia de las figuras parentales o de las experiencias difíciles que viven; desde esta perspectiva, los alumnos manifestarían esto en la relación que establecen con sus pares y sus profesores. Finalmente, otro factor identificado por los docentes es la "historia escolar", aludiendo a experiencias escolares anteriores positivas y negativas. Desde su perspectiva, sus vivencias anteriores influyen en el modo como enfrentan su vida escolar actual y los estilos relacionales que establecen con sus pares $\mathrm{y}$ adultos.

Una tercera agrupación de factores refieren a los que dependen de ellos mismos, estos pueden agruparse en: profesionales y personales. Los primeros relacionados con habilidades profesionales, estilos de metodología en el aula, dominio de grupo y su quehacer pedagógico en general. Desde su perspectiva, cuando un profesor posee estrategias metodológicas que permiten que todos los alumnos estén trabajando y teniendo un rol activo durante la clase, la aparición de situaciones conflictivas tienden a disminuir, en particular con aquellos alumnos que por sus características 
son complejos en su manejo. Adicionalmente, también dan importancia a la planificación del trabajo en aula:

“...la planificación, para mí es súper importante, decir ya primero les voy a pasar esto, después le voy a pasar lo siguiente, entonces una ya lleva, todo, todo planificado y planeado que es lo que uno va hacer, si no se producen los desórdenes y desmanes, al no llevar una planificación, por eso para mí es supremamente importante el mantener a las alumnas trabajando...” (E1).

Otra característica que dan importancia en lo profesional, es que conozcan bien a sus alumnos, es decir, sus rasgos personales, sus historias, situación vital y experiencias cotidianas. Uno explica: “el profesor debe saber todo lo que le pasa al niño, saber dónde vive, que problemas tiene, si vive con la mamá, si vive con el papá, saber todo... entonces así se involucra con el alumno" (E3). Desde la visión de los docentes, un mayor o menor conocimiento de los alumnos influye en la forma de relacionarse con ellos y orienta las estrategias de manejo disciplinario en lo individual y grupal.

Por otra parte, las variables personales, pueden categorizarse en: emocionales, comunicacionales y relacionas con su carga laboral. En las emocionales, por ejemplo, perciben que el nivel de estabilidad emocional que tenga el profesor en el día a día, influiría en la forma de relacionarse con sus alumnos y el modo de enfrentar los conflictos: “podís’ llegar un día enojá' a la sala y tu forma de relacionarte con las personas va a ser distinta a un día que llegues contenta, porque uno no se sustrae de las emociones en este caso" (E3)

A partir de las explicaciones de los profesores puede desprenderse que estas variables (alumno, institución y profesor) tendrían influencias reciprocas entre sí y la combinación de estos factores, generaría una influencia única y particular para cada establecimiento sobre su convivencia escolar.A partir de sus concepciones, es posible diferenciar tres grandes tipos de definiciones de convivencia escolar (CE), estas son: aquellas definidas desde una perspectiva conductual - normativa, otra relacional y vincular, y definiciones asociadas a valores como respeto y disciplina. Un primer ámbito de definiciones se puede denominar como conductual - normativo, siendo compartido por la mayor parte de los sujetos del estudio, quienes aluden a la $\mathrm{CE}$ como un conjunto de normas para la regulación conductual dentro de un contexto escolar y que a partir de ellas, se definiría lo esperado o no de los distintos comportamientos de quienes participen de ese contexto normado, una docente lo expresa como: "convivencia escolar es la forma en cómo se relacionan e interactúan los miembros de la comunidad educativa, con sus normas, bueno con también hay un manual de convivencia... con las normas que se establecen dentro...”.(E4).

Un segundo ámbito, alude a CE como un espacio en donde se dan un conjunto de relaciones y vínculos entre estamentos y personas que forman parte del establecimiento educacional. "las relaciones que se dan en las 
personas que actúan o que están dentro de un establecimiento, un colegio, un liceo, las relaciones entre todas las personas, no solamente entre alumnos, entre profesores y alumnos sino, que entre todos“(E3). Finalmente, en un tercer ámbito de definiciones, están aquellas concepciones de la CE como el ejercicio cotidiano de valores como el respeto y la obediencia, dado que esto estaría asociado con una mejor disciplina y en consecuencia, a una mejor convivencia. Varios docentes concuerdan que estos valores de algún modo se expresan en los contextos relacionales y con mayor motivo en un colegio. La siguiente cita da cuenta en parte de esto:

“eso de alguna manera siempre (valores) se va a reflejar en la convivencia diaria por mucho que uno trate a lo mejor de arreglar las cosas o de ser mejores, puede influir en algún momento negativamente en contra de esa convivencia, la falta de respeto por ejemplo...” (E1).

Por otra parte, se desprende de las explicaciones de los profesores, que la CE estaría caracterizada por ser dinámica y cambiante, dependiendo de las situaciones, de las personas y de los comportamientos grupales de los cursos, aspectos que se dan cotidianamente y que varían día a día.

"sabemos que la convivencia se hace día a día, no es algo que tu estableces en un momento y ahí queda para siempre, a veces falla y a veces está bien, es algo que todos los días va cambiando (E3)”.

Al respecto, los docentes expresan que no sólo cambia con frecuencia, sino que diariamente se va construyendo y modificando por la influencia de los distintos miembros del sistema. Desde esta concepción de convivencia escolar, los profesores señalan que desarrollan algunas estrategias disciplinarias, las que pueden diferenciarse en tradicionales y emergentes. Las tradicionales, serían aquellas que el profesor aplica independientemente de un curso, esto es, acciones disciplinarias que ejecuta como parte de sus estrategias para el manejo de los grupos y las emergentes, aquellas que el docente aplica según las características del curso, de acuerdo al contexto y una situación en particular, por ejemplo una profesora señala: “...nosotros vamos a actuar según los acontecimientos que ocurran...” (E2). Desde los docentes, el tipo de CE que se tenga en un curso determinado o en el establecimiento, va a llevar a que ellos tengan que adoptar un manejo con un énfasis en lo disciplinario-normativo y coherente con ello, una particular forma de enfrentar, manejar y resolver los conflictos que se le demanden.

La combinación e influencia de estos múltiples factores, facilitarían la aparición de consecuencias, como las explicaciones que los profesores realizan hacia el desorden u otras conductas disruptivas, algunas de estas se refieren a: a) las conductas disruptivas de los alumnos son algo natural, es decir, surgen espontáneamente como parte de sus conductas cotidianas; b) son una reproducción de modelos externos al establecimiento; c) formas de llamar la atención debido a sus dificultades; d) modos de defensa hostil aprendida desde el medio, como una forma de mostrarse ante los demás. En la siguiente cita se ve un ejemplo de estas explicaciones: 
"el desorden es como el diario vivir de ellos, porque en la casa son así, entonces uno tiene que, en la sala de clases luchar contra las costumbres que tienen en la calle y las costumbres que tienen en la casa” (E3).

A partir de estas explicaciones, los profesores justifican que necesitan flexibilizar en el manejo disciplinario, dadas las dificultades y carencias que viven sus alumnos y que se expresan en estos comportamientos.

“...les he prohibido que coman en la sala todo eso, pero de repente me cuestiono que a veces en la mañana a primera hora se están comiendo un sándwich, un yogurt y últimamente las estoy dejando, porque pienso que no han tomado desayuno...” (E2).

Otra consecuencia de las dinámicas de CE, es el mayor o menor desgaste docente que se produce en el equipo, como consecuencia de las estrategias que deben realizar para manejar la convivencia dentro de un grupo de curso, una profesora señala: "eso de estar llamando la atención o no, corrigiéndolo... eso desgasta más..... “(E3). Finalmente, a partir de sus discursos, se puede extraer que ellos visualizan algunas posibilidades de cambio, ya sea desde la perspectiva de la disciplina cotidiana y como resultado de un proceso formativo (a largo plazo). Los docentes explican que en la medida que los alumnos permiten la comunicación, siguen las normas establecidas, participan de la clase, y a la vez tienen buenos modelos de ejemplo (ellos como formadores), es posible que aparezcan otras alternativas de conducta, individuales y grupales. En cuanto al resultado como proceso formativo, manifiestan que existe un porcentaje importante de alumnos que al salir de cuarto medio, evidencian un cambio significativo.

\section{Figura $N^{\circ} 1$. Modelo: Construcciónn de Convivencia Escolar}

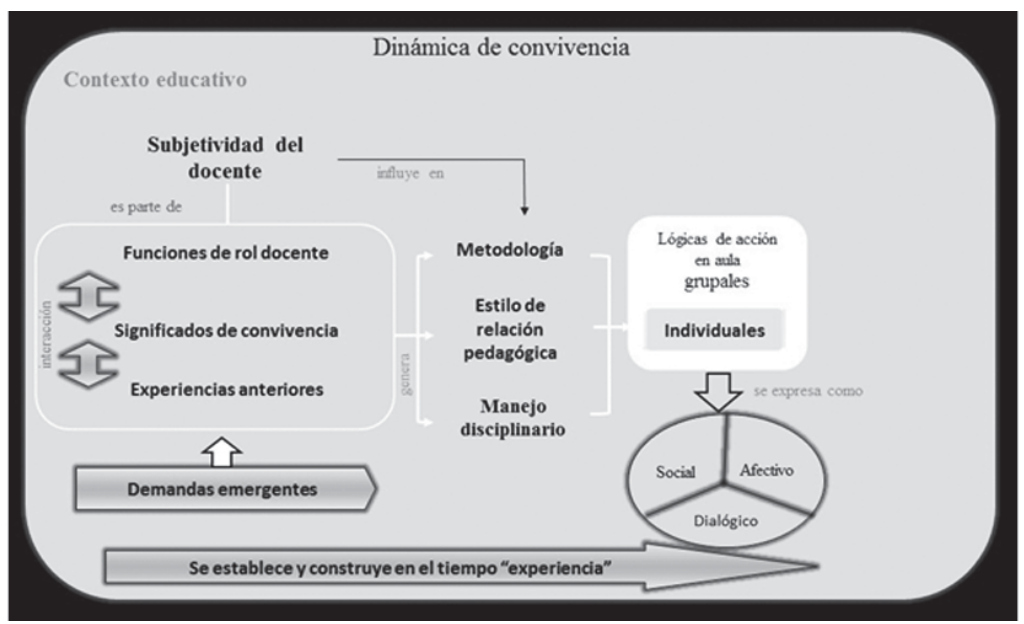




\section{Dinámicas de construcción de la convivencia escolar}

La codificación selectiva da cuenta de la dinámica de construcción cotidiana que se da en la convivencia escolar y en particular con lo que puede ocurrir a nivel de aula, a partir de la subjetivad del docente, considerado en el presente estudio desde las teorías subjetivas y el encuentro en el marco de la relación pedagógica con la subjetividad del alumno. Desde la perspectiva del docente, está compuesta por tres importantes componentes que representan las teorías subjetivas fundamentales, el primero se refiere al tipo de rol docente que el profesional asume y adopta, explicado en el modelo de la diversidad de rol con que muchos justifican y explican su responsabilidad con sus alumnos, esto podría plantearse interpretativamente reconstruyendo una teoría subjetiva, planteada como : "los alumnos con los cuales trabajamos provienen de contextos limitados y carentes, entonces debo ayudar a suplir dichas carencias a través de diversas acciones con el fin de educarlos -en el amplio sentido- y en algunos casos ayudarlos”. Esto tendría repercusiones de diferente índole: percepción de las necesidades del alumno, expectativas de resultado inmediato y futuro, visión de sus posibilidades educativas y capacidad de cambio y movilidad social.

Un segundo aspecto a la base de la subjetividad de los profesores, se refiere a sus concepciones respecto de la convivencia escolar y como la definen, dado que esto influye en sus percepciones respecto al tipo de convivencia que debe darse en el aula y la forma de construirla en los espacios cotidianos de interacción. Un tercer factor que interactúa con las nociones de rol docente y la concepción de convivencia escolar, tiene que ver con el peso subjetivo que ejercen las experiencias anteriores, dado que para los profesores gran parte de sus explicaciones las basan en las múltiples experiencias que han tenido que enfrentar con alumnos y diferentes grupos de curso, dándole una significativa relevancia a los momentos en que han tenido que responder ante conflictos, superar problemas de disciplina, enfrentarse con episodios complejos de interferencia durante las clases, entre otros, que en suma constituye su valorada experiencia profesional. subjetiva:

Este conjunto de factores podría reconstruirse en la siguiente teoría

"Los años que llevo como profesor municipal, me han demostrado que este tipo de alumnos con los cuales trabajamos, nos demandan que seamos más que un profesor que les enseña ciertos contenidos, sino alguien que se preocupe de ellos, que los acoja afectivamente, que los formen para la vida... y que tengamos esto en consideración para trabajar con ellos y entender sus conductas y reacciones”.

Con esta particular forma de interpretar lo que les ocurre, se podría plantear que al momento de enfrentar nuevas demandas emergentes propias de la cotidianidad de su quehacer; la observación, significación y respuesta de lo que acontece en el momento, se conjuga con los fenómenos 
interpretativos que explicábamos anteriormente, dando paso, a la confirmación de sus propias teorías ya estructuradas o generando una reacomodación cognitiva y probablemente también afectiva, acerca del grupo de alumnos o de uno de ellos en particular, con su consecuente efecto en la acción.

Esta conjugación de factores es dinámica, interactuando permanentemente tanto los procesos interpretativos instalados ya en el tiempo, por medio de lo que ellos denominan la "experiencia”, como los que están entrando en forma permanente a través de "nuevas experiencias". A partir de estas conjugaciones e interpretaciones de los profesores, se generarían tres tipos de consecuencias en sus acción: una relacionada con el tipo de metodología que implementan en el aula para llevar a cabo el aprendizaje de sus asignaturas, el tipo de relación pedagógica (profesor - alumno) que establecen y el tipo de manejo disciplinario que aplican.

La acción metodológica, se refiere a la forma de trabajar el currículum en el aula. A partir de sus discursos se desprende la idea, de que ellos muchas veces modifican la forma de enseñar y de generar el contexto de aprendizaje a partir de las características que observan en los cursos y sus necesidades, con ello, evaluarían la forma de entregar los contenidos, el tipo de actividades que se puede realizar con esos grupos e incluso el nivel de profundidad que se puede alcanzar en el tratamiento de materias. Una segunda consecuencia se refiere a la relación profesor - alumno que el profesor concibe tanto a nivel grupal como de las percepciones que tiene de cada alumno, especialmente en relación a sus necesidades afectivas, y como esto finalmente influye en el tipo de relación que busca establecer y mantener. Los profesores explicitan que es importante que ellos conozcan a sus alumnos, acerca de sus dificultades, historias personales y contextos familiares de los cuales provienen, esto les permite definir la forma de actuar con ellos, el tipo de relación que se puede tener, la forma de comunicarse y en términos generales el tipo de vínculos que se puede llegar a establecer con cada uno de ellos. Una tercera consecuencia en la acción, se refiere al manejo disciplinario que el profesor busca aplicar. Los docentes explican que cada grupo de estudiantes es diferente al otro y por lo tanto, deben tener procedimientos de disciplina distintos según sus características y edades, e incluso según las situaciones y dificultades que emerjan cotidianamente. Explican que según el tipo de rol que les toque enfrentar, las necesidades que observen de sus alumnos y su grupo, facilita que en ocasiones deban flexibilizar su postura disciplinaria, disminuir su exigencia en términos conductuales y dejar en segundo plano los contenidos de aprendizaje, para otorgar el tiempo necesario para conversar con ellos, comprender sus problemáticas y aconsejarlos (orientarlos) en sus acciones.

Así, y desde las explicaciones de los docentes, estas miradas interpretativas tendrían una consecuencia que se vería expresada en las dimensiones afectiva, social y dialógica. A nivel afectivo se expresan tipos de estados de ánimo y emoción del docente; en su expresión social, surge una dinámica particular de relación interpersonal a nivel grupal; y en términos dialógicos se desarrollan e instalan estilos de comunicación entre los 
actores que participan en el aula y actos lingüísticos recurrentes, que definen y dan cuenta del modo de estar en ese lugar.

\section{Discusión}

Presentamos en primer lugar una síntesis interpretativa de los resultados, para luego pasar a discutirlos desde los objetivos de nuestro estudio. Los resultados de este estudio, dan cuenta de las teorías subjetivas del profesor en cuanto a su rol en la convivencia escolar, el cual es concebido como flexible, ya que cambiaría según las características, necesidades y dificultades de los alumnos con los que trabajan. Los docentes dan mayor relevancia a funciones que impliquen desde sus significados "rescatar” a los alumnos, dándoles una educación para la vida, el trabajo y constituyéndose en un soporte en lo afectivo, roles que estarían por sobre objetivos académicos. Esto implicaría que adoptan un rol protector en la relación con sus alumnos, lo que en algunos casos pasa a ser prácticamente asistencial por el tipo de necesidades que intentan cubrir.

Ala vez, podríamos indicar que el espacio del aula es el lugar donde se produce el encuentro intersubjetivo, entre las concepciones interpretativas-explicativas del docente y las del alumno, permitiendo esta conjugación cierto tipo de lógica de acción que influyen en la negociación de significados entre ambos (Bardisa, 2009; Nail, Muñoz y Gajardo, 2013). En el caso de los alumnos, podrían estar mediadas por la influencia que puede ejercer el grupo de pares, por sus propias formas de interpretar y por las lógicas de acción que permite el propio docente.

Como consecuencia de lo anterior se va desarrollando un clima de aula, ya que es el espacio donde convergen y se expresan estos elementos comentados. Se entiende el clima de aula entonces, como la suma entre la subjetividad del profesor, la consecuencia en sus actos, la subjetividad del alumno y las lógicas de acción que de este encuentro se derivan, constituyendo una forma de organizarse, relacionarse, expresarse, en síntesis convivir. El clima de aula tendría una repercusión o influencia en la convivencia escolar superior, dado que es la representación micro o un subsistema, dentro de lo que acontece a un nivel superior o sistema mayor, representado por la escuela en su conjunto.

En definitiva, la construcción de la convivencia se constituye en este proceso constante de instalación y reconstrucción, donde el profesor se transforma en un lector activo, interpretativo de lo que observa del clima de aula y la convivencia escolar, retroalimentando en forma constante sus formas de interpretación (teorías subjetivas) y a la vez, constituyéndolo en lo que éstos denominan como "su experiencia”, la que ciertamente se va desarrollando con el pasar del tiempo y con las múltiples experiencias cotidianas que les acontecen. 
Un objetivo del estudio fue comprender desde la perspectiva del docente el tipo de relación profesor-alumno que se genera al interior del aula. Para los docentes existe un importante compromiso de tipo afectivo, que se caracteriza por significados asociados a la percepción de que los alumnos tienen importantes necesidades de afecto, pocas oportunidades y bajas expectativas en términos educacionales. Esta visión del estudiante se ha construido a partir de su experiencia, no formal, en el trato directo con los alumnos, y que se vuelve a replicar en cada relación nueva que establecen tanto en el plano individual como grupal.

Otro objetivo consistió en conocer las teorías subjetivas acerca de los procesos formativos, al respecto es posible plantear que el tipo de formación que aparece en sus discursos está asociada a una formación hacia la vida, que les permita mantenerse en la sociedad, trabajar, formar familia y ser buenos ciudadanos. En este contexto, visualizan además valores en formación vinculados fundamentalmente al "respeto" como un ideal a alcanzar, el que según su visión, estaría asociado fundamentalmente al seguimiento de instrucciones (obediencia) y una relación adecuada con la autoridad, siendo deseable que se muestren atentos y bien adaptados a distintos contextos. Así también, en un segundo rango de valoración aparecen otros valores, asociados a la vida laboral, como la puntualidad y responsabilidad en la tarea. Estas concepciones tienen como implícito un enfoque basado en la obediencia (Bugueño y Mena, 2008), denotando así una "concepción tradicional de convivencia escolar" (Banz, 2008) basada en el seguimiento y reproducción como fuentes de desarrollo, en oposición a una perspectiva que implique la formación socio-afectiva, desarrollo de competencias genéricas y la educación moral de los individuos (Hirmas y Carranza, 2008; Von Reininghaus, Castro y Frisancho 2013).

Siguiendo los planteamientos de que en el aula confluyen los procesos subjetivos de los profesores con los de los alumnos (Ortega, 2011), junto con el marco de significados que aporta el contexto en el cual se encuentran insertos, constituyendo así una dinámica constante y cambiante que se va re-construyendo en los encuentros sucesivos, definiendo lo "intersubjetivo" desde el punto de vista de la convivencia, esto es: sus relaciones sociales, estilos comunicacionales y vinculares, modos de comportase y definir en lo implícito o explícito el marco de conductas posibles en esos espacios.

Esto da pie a que los profesores frente a problemáticas de los alumnos, dificultades de disciplina o situaciones emergentes, decidan ser lo suficientemente flexibles para dar cabida a dichas necesidades y priorizar el abordaje de temas diversos por sobre el tratamiento de contenidos de aprendizaje, siendo para ellos, más relevante conversar y tratar temas relacionados con la vida personal, abordar la necesidad de formar hábitos personales (orden, limpieza, responsabilidad, etc.), manejo de situaciones de conflicto y problemas de conducta que afectan la disciplina, ya que consideran que esto, es una necesidad de mayor prioridad y más básica, que el trabajo específico de una asignatura.Tomando como premisa de que lo social en 
general y en particular a convivir "se aprende” (Maldonado, 2004; Mena, Becerra y Castro, 2011), es interesante plantear una postura crítica y reflexiva respecto del tipo de aprendizajes que se están generando a partir de estas concepciones y construcciones intersubjetivas y de su influencia que esto conlleva en los procesos de formación de habilidades socio afectivas y éticas, de alumnos que asisten a estos establecimientos.

Finalmente, a partir del análisis de los resultados, se podría hipotetizar que si se busca mejorar la convivencia y por ende la cultura escolar, esto implicaría un proceso de cambio de las concepciones docentes acerca de los alumnos y de los procesos formativos en que participan al interior de un establecimiento escolar. Lo anterior desde una interacción con la implementación progresiva de prácticas diferentes en el sistema escolar. Para de este modo, facilitar desde su rol las habilidades socio-afectivas, potenciar la autonomía, promover el emprendimiento, generar expectativas educacionales como parte de la movilidad social y de modo importante, fortalecer el desarrollo moral y ético. En definitiva, promover una educación para la convivencia, la paz, la formación democrática en mundo diverso y cambiante (Hirmas y Carranza, 2008). Esto último, abre también un campo de acción que hay que seguir trabajando e investigando, referido por una parte a la definición del rol docente en la educación pública, el desarrollo de competencias docentes, su formación inicial y el fortalecimiento de espacios de reflexión, respecto de la formación y la convivencia como aprendizaje, temas que requieren ser trabajados en diversos contextos educativos y de formación profesional. 


\section{Nota}

${ }^{1}$ Los resultados presentados en este artículo se basan en la investigación del primer autor para optar al grado de Magíster en Psicología, mención Psicología Educacional, de la Universidad de La Serena, Chile. Los autores desean agradecer a los profesores que participaron en el estudio y a las instituciones que los respaldaron. 


\section{Bibliografía}

Aguilar, J. (2010). El docente y la aplicación de estrategias de enseñanza. Revista de Investigación e Innovación Educativa para el desarrollo y la formación profesional(1),159-182.

Ascorra, P., Arias, H. y Graff, C. (2003), La escuela como contexto de contención social y afectiva. Revista Enfoques Educacionales, 5 (1) 117 - 135. Recuperado de http://www.facso.uchile.cl/publicaciones/ enfoques/07/Ascorra_Arias_Graff_EscuelaContencion SocialAfectiva.pdf

Aristeguí, R., Bazán, D., Leiva, J.,López, R., Muñoz, B., y Ruz, J. (2005). Hacia una pedagogía de la convivencia. Revista Psykhe,14 (1),137 150. doi: https://dx.doi.org/10.4067/S0718-22282005000100011

Banz, C. (2008). Convivencia escolar. Valoras UC.Recuperado dehttp:// portales.mineduc.cl/usuarios/convivencia_escolar/doc/ 201103041353340.Valoras_UC_Convivencia_Escolar.pdf

Bardisa, T. (2009). Análisis y resultados de la construcción del sistema de convivencia escolar,Educar (43) 95-127. Recuperado de http:// ddd.uab.cat/pub/educar/0211819Xn43p95.pdf

Botella, L. (2001). El ser humano como constructor de conocimiento: el desarrollo de las teorías científicas y las teorías personales.Recuperado de http://fpce.blanquerna.edu/ constructivisme/Papers/Conocimiento.htm>.

Briones, G. (1989). Métodos y técnicas avanzadas de investigación aplicadas a la educación y a las ciencias sociales. Bogotá. Colombia: ICEFES - PIIE

Bugueño, X. y Mena, I. (2008).El sentido del reglamento de convivencia: Lógicas y sugerencias para su construcción. Recuperado de http:// valoras.uc.cl/wp-content/uploads/2010/10/reglamento_conv.pdf

Castro, P. y Cárcamo, R. (2012). Cambio de teorías subjetivas de profesores respecto a la educación en valoresSubjetividad y procesos cognitivos, 16 (1) 17-42. Recuperado de http://www.scielo.org.ar/ pdf/spc/v16n1/v16n1a01.pdf

Catalán, J. (2010). Teorías subjetivas. Aspectos teóricos y prácticos.La Serena, Chile: Editorial Universidad de La Serena.

Ídem (2014). Elaboración de teorías subjetivas de preescolares en el aula. Polis Revista Latinoamericana13 (37), 41-62.Recuperado de https:/ /polis.revues.org/9688 
Ministerio de Educación (2005).Marco curricular de la enseñanza media. Objetivos fundamentales y contenidos mínimos obligatorios de la educación media. Santiago, Chile: Gobierno de Chile .

Flick, U. (2009). An introduction to qualitative research.London, England: Sage.

Flick, U. (ed,), (1992). La perception quotidienne de la santé et de la maladie, Théories subjectives et repréntations sociales. Paris, France : L'Hartmann.

Flores, A., Lagos, A., Porra, C., y Narea, M. (2012). "La auto-e?cacia docente para la resolución de con?ictos entre profesores,” Educación y Educadores, 15() 265-288. Recuperado de http://www.redalyc.org/ articulo.oa?id=83424870007

Gergen, K. (2006). El yo saturado, Dilemas de identidad en el mundo moderno, Madrid, España: Editorial Paidós Ibérica

Gómez, L. (2008).Las teorías implícitas de los profesores y sus acciones en el aula. Revista Electrónica Sinéctica() 1-14. Recuperado de http:// www.redalyc.org/articulo.oa?id=99819167005

Gómez, L. (2003).Las teorías implícitas de los profesores y sus acciones en el aula. Ponencia presentada en el VII Congreso Nacional de Investigación Educativa en Guadalajara, México.

Glaser, B. y Strauss, A. (1967). The discovery of grounded theory, Strategies for qualitative research. San Francisco, United States: Aldine de Gruyter.

Hargreaves, D. (1979).Las relaciones interpersonales en la educación. Madrid, España: Narcea.

Hirmas, C. y Carranza, G. (2009). Matriz de indicadores sobre convivencia democrática y cultura de paz en la escuela. En UNESCO, Documento de III Jornadas de Cooperación Iberoamericana sobre Educación para la Paz, la convivencia democrática y los derechos humanos, pp. 56 - 136, Santiago, Chile: Salesianos Impresores.

Ianni, N. (2003). Convivencia escolar. Una tarea posible, necesaria y complejas. Monografías virtuales Ciudadanía, democracia y valores en sociedades plurales, (2). Recuperado de http.//www.oei.es/valores2/ monografias/monografia02/reflexion02.htm

Kincheloe, J. (2001). Hacia una revisión crítica del pensamiento docente. Barcelona, España: Editorial Octaedro.

Krause, M. (1995). La investigación cualitativa.Un campo de posibilidades 
y desafíos.Revista temas de educación (7) 19-39. Recuperado de https://investiga-aprende-2.wikispaces.com/file/view/Inv-cualitatKrause.pdf

Krause, M. (2005), Psicoterapia y cambio. Una mirada desde la subjetividad. Santiago, Chile: Ediciones Universidad Católica

Jiménez, A. (2005). Las teorías implícitas del profesorado sobre los distintos ámbitos del proceso de enseñanza-aprendizaje: la interacción profesor-alumno en los tres niveles educativos. Revista Qurriculum (18),211-236. Recuperado de http://publica.webs.ull.es/upload/ REV\%20QURRICULUM/18\%20-\%202005/10\%20(Ana\% 20Beatriz\%20Jim\%C3\%A9nez\%20Llanos).pdf

Maldonado, H. (2004). Convivencia escolar, Ensayos y experiencias. Buenos Aires, Argentina: Lugar Editorial.

Mena, I., Romagnoli, C. y Valdés, A. (2006). Convivencia escolar. Santiago, Chile: Documento ValorasUC.

Mena, I., Becerra, S. y Castro, P. (2011).Gestión de la convivencia escolar en Chile, Problemáticas, anhelos y desafíos. EnCatalán, J. (ed.), Psicología Educacional Proponiendo rumbos, problemática y aportaciones, 81-112.La Serena, Chile: Editorial Universidad de La Serena.

Nail, O., Muñoz, M. yGajardo, J. (2013).Principios orientadores de la convivencia en el aula: Una estrategia de reflexión colectiva. Educação e Pesquisa, 39(2), 367-385. https://dx.doi.org/10.1590/S151797022013000200006

Ortega, M. (2011). Construcción de significados y aprendizaje situado en un contexto de intersubjetividad en un programa de formación de licenciados en intervención educativa. XI Congreso Nacional de Investigación Educativa. UNAM, México DF, México.

Pontificia Universidad Católica de Chile (2006). Síntesis enfoques didácticos, fundamentos y características, Curso de Didáctica General de la Facultad de educación de la Pontificia Universidad Católica, Santiago, Chile.

Rodrigo, M.J., Rodríguez, A. y Marrero, J. (1993).Las teorías implícitas. Una aproximaciónal conocimiento cotidiano. Madrid, España: Visor Distribuciones.

Stake, R. (1998). Investigación con estudio de casos. Madrid, España: Ediciones Morata.

Taylor, S. J. y Bogdan, R. (1996). Introducción a los métodos cualitativos de investigación. Madrid, España: Paidós Básica. 
Vasilachis de Gialdino, I. (1992).Métodos cualitativos I. Los problemas teóricos metodológicos.Buenos Aires, Argentina: Centro Editor de América Latina.

Vásquez, A. (2008). Zigmunt Bauman, Modernidad líquida y fragilidad humana. Nómadas, Revista Crítica de ciencias Sociales y Jurídicas(19). Recuperado de http//pendientedemigracion,ucm,es/info/ nomadas/19/avrocca2,pdf

Von Reininghaus, G., Castro, P. y Frisancho, S. (2013). School violence, Subjective theories of academic advisory board members from six chilean schools. Interdisciplinaria 30 (2) 219-234, Recuperado de $70272013000200003 y l n g=$ esynrm=iso $>$. ISSN 1668-7027

Recibido: 24.09.14

Aceptado:15.05.15 